\title{
Use of transesophageal echocardiography during cesarean section in a parturient with inferior vena cava thrombosis
}

\author{
Huynh PM*, Rayan H, Huh M, Tran Q and Kakazu C \\ Department of Anesthesiology, Harbor-UCLA Medical Center, 1000 West Carson Street, Torrance, California, USA
}

\begin{abstract}
The use of transesophageal echocardiography in obstetric anesthesia is uncommon and rarely reported during cesarean section. To our knowledge, this is the first report of its use for intraoperative surveillance of a tumor thrombus that has invaded over $90 \%$ of the inferior vena cava and was at risk of embolizing in a patient undergoing a cesarean section. We decided to use transesophageal echocardiography in this case to directly observe the IVC tumor which would allow for early intervention and to potentially avoid impending cardiovascular collapse. This case also reports a rare tumor that was incidentally found in late pregnancy which made a safe delivery to be very challenging.
\end{abstract}

\section{Introduction}

Transesophageal echocardiography (TEE) was developed in the 1960s and found its way into cardiac anesthesiology for intraoperative surveillance during cardiopulmonary bypass, heart valve management, and other cardiac procedures. While it is routinely used in cardiothoracic surgeries, TEE is uncommonly used in obstetric anesthesia. We report the use of TEE in a patient with Ewing's sarcoma of the kidney undergoing cesarean section. The sarcoma had extended into the inferior vena cava and caused a tumor thrombosis which was at risk of embolizing and leading to cardiovascular collapse. TEE was used for real-time intraoperative surveillance of the tumor thrombus. It was also used to help differentiate possible causes of cardiovascular collapse such as volume status due to bleeding and thrombus migration to the pulmonary artery. We valued the use of transesophageal echocardiography in this case report because it could visually confirm tumor thrombus migration and quickly narrow the differential diagnosis of hemodynamic instability. The patient provided written permission for publication of the report.

\section{Case presentation}

A 16 year old G1P0 parturient at 37 weeks of pregnancy presented from an outside hospital for higher level of care because of a recently diagnosed renal mass. She endorsed a five month history of left upper quadrant discomfort that was routinely dismissed as a pregnancyrelated problem. Eventually, an MRI from an outside hospital performed at 27 weeks of gestation revealed a large mass arising from her left kidney. Further imaging with ultrasound measured it to be $21 \mathrm{~cm} \mathrm{x} 15 \mathrm{~cm}$ in size. Following a multidisciplinary discussion, a presumptive diagnosis of renal cell carcinoma was made based on its rapid growth during pregnancy and the patient's age. Biopsy for a definitive diagnosis was deemed unfeasible because of her late stage of pregnancy. Her management was further complicated by the discovery of a large inferior vena cava tumor thrombus in the suprahepatic region that was not amenable to inferior vena cava (IVC) filter placement (Figure 1). Post-delivery auto transfusion presented a concern for the tumor thrombus to migrate centrally with a potential risk for sudden cardiovascular collapse. After a multidisciplinary meeting involving the obstetric, urologic, and cardiothoracic, neonatology, obstetric anesthesiology, and cardiac anesthesiology teams, it was concluded that a cesarean section was a safer option given the risk of an unexpected peripartum complication or emergency. Additionally, the team felt optimal management of the renal mass should proceed several weeks after the cesarean delivery when her cardiovascular status had returned to baseline. Continued discussions between different teams and the patient concluded a cesarean section with eventual removal of the tumor to be the safer option.

Preoperatively, the patient was premedicated with $30 \mathrm{ml}$ of oral sodium citrate. $1 \mathrm{mg}$ of intravenous midazolam was given as

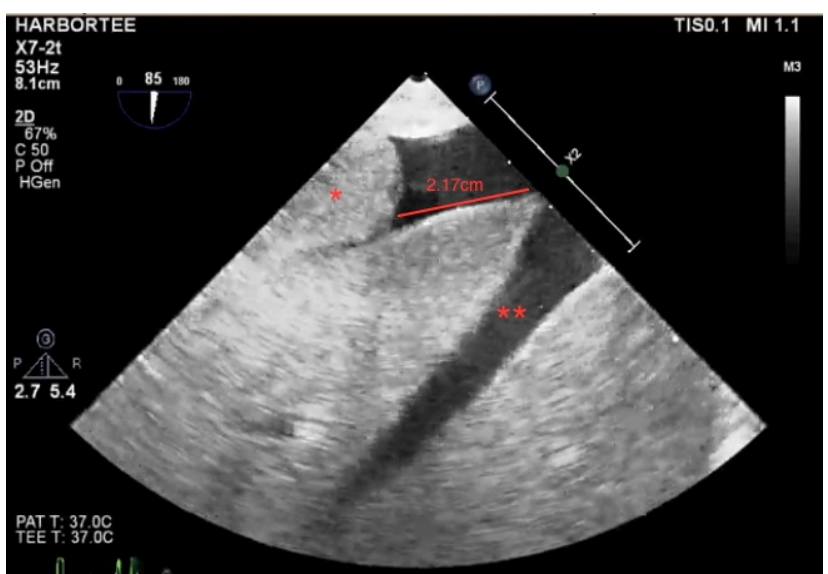

Figure 1. TEE long-axis view of the liver showing the tumor thrombus $\left(^{*}\right)$ located $2.17 \mathrm{~cm}$ away from the hepatic vein $(* *)$ and IVC junction.

Correspondence to: Huynh PM, Department of Anesthesiology, Harbor-UCLA Medical Center, 1000 West Carson Street, Torrance, California, USA, Tel: (310)222-5067; E-Mail: For.Peter.Huynh@gmail.com

Received: August 14, 2017; Accepted: October 26, 2017; Published: November 03, 2017 
patient was extremely nervous and hyperventilating which could cause vasoconstriction of the umbilical vessels. Her baseline blood pressure was $118 / 80 \mathrm{mmHg}$ with a heart rate of 97 beats per minute. Her presenting hemoglobin was $9.2 \mathrm{~g} / \mathrm{dL}$ despite being on prenatal vitamins and iron. Our plan was to perform a spinal anesthetic with preservative-free (PF) morphine followed by general anesthesia. Spinal anesthesia was used for postoperative pain control. General anesthesia was used to secure the airway in the event of bleeding, tumor embolism, or need for possible cardiopulmonary bypass. The spinal anesthetic was performed at the fourth and fifth lumbar vertebrae in the sitting position using a 25 gauge Whitacre needle to deliver $20 \mu \mathrm{g}$ of fentanyl and $0.2 \mathrm{mg}$ of PF morphine for postop pain control. The obstetrical team did not consider intraoperative fetal heart monitoring necessary during or after the neuraxial block. We did not feel it was necessary to check for sensory level as spinal narcotics were used for pain control. Patient was placed in a supine position with a left lateral tilt and preoxygenated with $100 \%$ oxygen. A rapid sequence induction was performed with cricoid pressure using $2 \mathrm{mg} / \mathrm{kg}$ of propofol and $2 \mathrm{mg} / \mathrm{kg}$ of succinylcholine. A grade I laryngoscopic view was obtained with a size three Macintosh blade, and the airway was secured atraumatically with a size $7.0 \mathrm{~mm}$ endotracheal tube. General anesthesia was maintained with $100 \% \mathrm{FiO} 2,0.2 \mathrm{MAC}$ of sevoflurane, a propofol infusion at $50 \mu \mathrm{g} / \mathrm{kg} / \mathrm{min}$, and a remifentanil infusion at $0.2 \mu \mathrm{g} / \mathrm{kg} / \mathrm{min}$. In the case of tumor thrombus migration, we maintained $100 \% \mathrm{FiO} 2$ throughout the case as an additional measure of safety to allow more time if cardiopulmonary bypass became necessary. Given our concern for possible cardiovascular instability, we placed a left radial arterial line and an 8.0 French double lumen central line in the right internal jugular vein under ultrasound guidance after induction.

Prior to surgical incision, a baseline TEE revealed the left ventricular ejection fraction to be $55 \%$, and the thrombus size to be $33 \mathrm{~mm} \times 37$ $\mathrm{mm}$ in its short axis which occupied over $95 \%$ of the IVC (Figure 2). It extended proximally $2.5 \mathrm{~cm}$ distal to the IVC/hepatic vein junction. We visualized small slit-like blood flow on color Doppler seen flowing around the tumor thrombus in the IVC. To avoid disturbing the tumor and tumor thrombus, the urologist performed a vertical midline abdominal incision from the pubic symphysis to the xiphoid process. At this point, the obstetrical team proceeded with the cesarean section with a low transverse uterine incision. After eleven minutes, the neonate was delivered with APGAR scores of 2, 5, and 8 at 1 minute, 5 minutes, and 10 minutes respectively. The patient received a 3 unit bolus and 37 unit

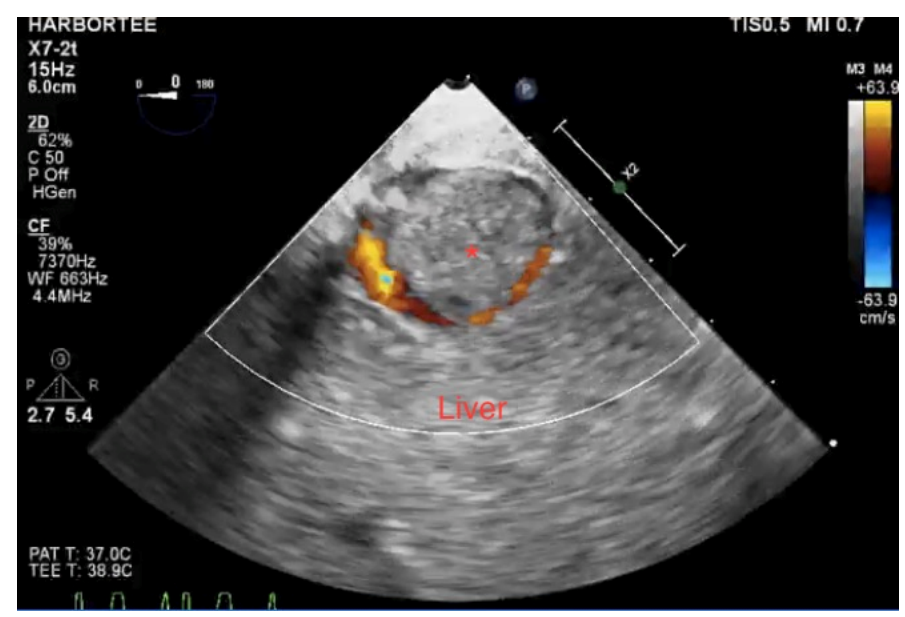

Figure 2. TEE short-axis view of the liver showing the tumor thrombus $(*)$ measuring $33 \mathrm{~mm} \times 37 \mathrm{~mm}$ occupying over $95 \%$ of the IVC. Color Doppler shows a small slit-like blood flow around the tumor thrombus in the IVC. infusion of oxytocin and $0.2 \mathrm{mg}$ of intramuscular methylergonovine maleate. Throughout the case, she received $1500 \mathrm{ml}$ of crystalloid, 4 units of packed red blood cells, and 2 units of fresh frozen plasma. The estimated blood loss was 2 liters and the urine output was $450 \mathrm{ml}$. An arterial blood gas after delivery revealed the following values: $\mathrm{pH} 7.3$, PCO2 $43 \mathrm{mmHg}$, PO2 $390 \mathrm{mmHg}$, Na $136 \mathrm{mEq} / \mathrm{L}, \mathrm{K} 4.3 \mathrm{mEq} / \mathrm{L}$, Ca $1.03 \mathrm{mmol} / \mathrm{L}$, glucose $199 \mathrm{mg} / \mathrm{dL}, \mathrm{Hgb} 8 \mathrm{~g} / \mathrm{dL}, \mathrm{HCO} 321.2 \mathrm{mEq} / \mathrm{L}, \mathrm{BE}$ -4.9 , and $\mathrm{SaO} 2100 \%$. Of concern was the significant increase in cardiac output and auto-transfusion after delivery of the neonate which could cause tumor dislodgement. Surveillance of volume status and tumor thrombus with TEE was performed throughout the entire surgery by the cardiac anesthesiology team. It was noted that the tumor thrombus remained unchanged in position and morphology throughout the case. A biopsy of the renal mass following the cesarean section later revealed it to be Ewing's sarcoma. The endotracheal tube was removed in the operating room and the patient was transported to the intensive care unit for postoperative care. A Swan-Ganz was used postoperively to monitor pulmonary artery pressure for detection of the tumor thrombus. The patient was in the intensive care unit for 24 hours and was eventually downgraded to a monitored bed. On postop day one, 5000 units of heparin were given subcutaneously every 8 hours for thromboprophylaxis. A follow up transthoracic echocardiogram was later performed and the findings were unchanged.

\section{Discussion}

Primary Ewing's sarcoma of the kidney is a rare tumor in adults that was first described in 1975 and has since been sporadically documented. It is believed renal Ewing's sarcoma originates from neural cells that invaginate into the kidney during development. Other authors theorize embryonic neural crest cells migrate into the kidney and undergo tumorigenesis [1]. After review of the literature, we found one case in which the tumor invaded the IVC. Under the impression that it was renal cell carcinoma, a left radical nephrectomy with partial replacement of the IVC using a bovine pericardial patch was performed [2]. There is one other case report from the literature in which a pregnant woman with Ewing's sarcoma had extension into the IVC. However, it is unclear whether she underwent a cesarean section [3]. Aside from this, we are unaware of any cases which involve a parturient with Ewing's sarcoma that extends into the IVC and is further complicated by a large thrombus.

There are a number of reports regarding renal cell carcinoma and IVC tumor thrombus management; however they are not complicated by a gravid uterus, do not mention the size of the tumor thrombus, and involve renal cell carcinoma instead of Ewing's sarcoma [4-6]. Nagy and Gyurkovics et al. describe a male patient with renal cell carcinoma who underwent right nephrectomy. The case was complicated by the discovery of an IVC tumor thrombus 6 months postoperatively which was removed with a balloon catheter via the internal jugular vein. Fukazawa and Gologorsky et al. explain the benefits of using TEE in the management of level 3 (intrahepatic IVC but below hepatic veins) and level 4 (above level 3) IVC thrombi in the setting of renal cell carcinoma but have no mention of patient gender nor indication of thrombus size. Lastly, Oikawa and Shimazui et al. investigated the benefits of TEE in IVC tumor removal with balloon catheters in patients with renal cell carcinoma. The only female in their five cases did not have a gravid uterus to complicate her presentation and only primary tumor sizes, not tumor thrombus sizes, were included. Intraoperative TEE in these previously mentioned reports was shown to be beneficial for managing IVC tumor thrombi in patients with renal cell carcinoma. However the available literature focuses on removing the thrombi 
with the assistance with TEE instead of pure surveillance. We have been unable to find other case reports describing a parturient at full term pregnancy complicated by a large right-sided Ewing's sarcoma and tumor thrombus with almost $100 \%$ IVC occlusion. We believe the nearly complete IVC occlusion, risk of tumor migration, and inability to safely manage the thrombus with an IVC filter or thrombectomy required intraoperative TEE for surveillance. Additionally, planning to remove the tumor in a separate operation after the patient's hemodynamic status had returned to baseline was another unique aspect of her anesthetic management.

This particular case presented several challenges to the anesthesiology team because of the size of the tumor and presence of a large tumor thrombus in the IVC. Potential lethal complications included a tumor mass effect on the IVC that may have impeded venous return and potential embolization of the thrombus to the pulmonary circulation leading to complete circulatory collapse. Given her unique presentation, general anesthesia was planned to facilitate intraoperative TEE and the theatre was set up for possible cardiopulmonary bypass in the event of a cardiovascular catastrophe secondary to massive thromboembolism. The surgery was performed in a cardiac operating room with the cardiopulmonary bypass machine, perfusionist, and cardiothoracic surgeon ready for immediate intervention.

We focused on minimizing sympathetic effects with intrathecal narcotics and judicious use of intravenous medications to maintain her blood pressure and heart rate within $20 \%$ of her baseline. We selected a balanced anesthetic technique with propofol, remifentanil, and sevoflurane to allow for greater control over uterine tone as opposed to sevoflurane alone. Additionally we used remifentanil to attenuate the maternal hemodynamic response to cesarean section [7]. It is possible remifentanil may have contributed to the initial depressed APGAR scores because it may cause transient but significant neonatal respiratory depression [7]. However, there is conflicting information in the available literature stating remifentanil is safe and does not cause neonatal respiratory depression [8-10]. It is difficult to exclude remifentanil as a contributing factor to the initial APGAR scores but we believe surgical, general anesthetic, and maternal aspects may have influenced the neonate's initial presentation. Given the patient's unique situation, potential mass effect on the IVC, and inability to safely remove the IVC tumor thrombus preoperatively, intraoperative TEE surveillance was a powerful tool to assess the status of the thrombus and to quickly predict the need for cardiothoracic and vascular surgery in the patient's management. Fortunately, the position of the tumor thrombus did not change. However, if it did, we believe TEE would be a vital tool in detecting early migration of the thrombus and allow for immediate intervention by the cardiothoracic team.

\section{References}

1. Hakky TS, Gonzalvo AA, Lockhart JL, Rodriguez AR (2013) Primary Ewing sarcoma of the kidney: a symptomatic presentation and review of the literature. Therapeutic Advances in Urology 5: 153-159. [Crossref]

2. Nam JK (2015) Primary Ewing Sarcoma of the Kidney with Inferior Vena Cava Invasion. Med Surg Urol 4: 146. [Crossref]

3. Ding Y, Huang Z, Ding Y, Jia Z, Gu C et al. (2016) Primary Ewing's Sarcoma/ Primitive Neuroectodermal Tumor of Kidney with Caval Involvement in a Pregnant Woman. Urol Int 97: 365-368. [Crossref]

4. Nagy Z, Gyurkovics E, Pajor P, Tarjányi M, Szijártó A et al. (2015) New method for treatment of inferior vena cava tumor thrombus - case study. Croatian Medical Journal 56: 139-144. [Crossref]

5. Fukazawa K, Gologorsky E, Naguit K, Pretto EA Jr, Salerno TA et al. (2014) Invasive renal cell carcinoma with inferior vena cava tumor thrombus: cardiac anesthesia in liver transplant settings. J Cardiothorac Vasc Anesth 28: 640-646. [Crossref]

6. Oikawa T, Shimazui T, Johraku A, Kihara S, Tsukamoto S et al. (2004) Intraoperative transesophageal echocardiography for inferior vena caval tumor thrombus in renal cell carcinoma. Int J Urol 11: 189-192. [Crossref]

7. Draisci G, Valente A, Suppa E, Frassanito L, Pinto R et al. (2008) Remifentanil for cesarean section under general anesthesia: effects on maternal stress hormone secretion and neonatal well-being: a randomized trial. Int $J$ of Obstetric Anes 17: 130-136. [Crossref]

8. Kan RE, Hughes SC, Rosen MA, Kessin C, Preston PG (1998) Intravenous Remifentanil, Placental Transfer, Maternal and Neonatal Effects. Anesthesiology 88: 1467-74. [Crossref]

9. Shaylor R, Ginosar Y, Avidan A, Eventov-Friedman S, Amison N et al. (2016) Predelivery remifentanil infusion for placenta accreta cesarean delivery under general anesthesia: an observational study. The Journal of Maternal-Fetal \& Neonatal Medicine 29. [Crossref]

10. Behdad S, Ayatollahi V, Harrazi H, Nazemian N, Heiranizadeh N et al. (2013) Remifentanil at induction of general anesthesia for cesarean section: Double blind, randomized clinical trial. Colombia MédicA. CM 44: 87-91. [Crossref]

Copyright: (C2017 Huynh PM. This is an open-access article distributed under the terms of the Creative Commons Attribution License, which permits unrestricted use, distribution, and reproduction in any medium, provided the original author and source are credited. 